\title{
Smartphone-based National Hearing Test Launched in South Africa
}

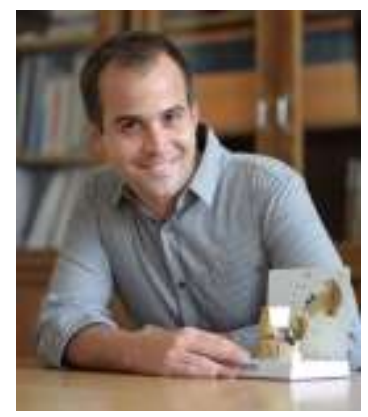

De Wet Swanepoel, PhD

1. Department of Speech-Language Pathology and Audiology, University of Pretoria, Pretoria, South Africa

2. Ear Science Institute Australia, Subiaco, Australia

3. Ear Sciences Centre, School of Surgery, The University of Western Australia, Nedlands, Australia

The hearZA App - South Africa's National Hearing Test - was launched on World Hearing Day 3 March 2016 (www.hearZA.co.za). More than 3000 people tested their hearing in the first 24 hours on iOS and Android versions of this South African English digits-in-noise test developed and validated at the University of Pretoria (Potgieter et al, 2016). The digits-in-noise test, which is self-administered, is quick and provides a valuable indication of real-life hearing ability - understanding speechin-noise.

Despite landline based digits-in-noise tests available in various countries around the world we set our sights on a smartphone platform for two important reasons. Firstly, with only $10 \%$ of South African households having access to a landline, employing a smartphone-based test that has close to $80 \%$ household penetration, was an obvious choice (STATSSA General Household Survey, 2013; Ericsson, 2015). Secondly, we wanted to leverage a smartphone platform for all the many additional possibilities it offers beyond detection of a hearing problem.

Our aim was therefore to have a 1) strategic public awareness tool for; 2) accurate detection of hearing loss that; 3 ) allows for personalized hearing health tracking; 4) in-app decision support and; 5) a referral network linking persons to their closest hearing health providers. 


\section{Public awareness tool}

Smartphones are an integral part of modern day living with health applications becoming central to their monitoring features. We wanted to capitalize on this trend to increase awareness of healthy hearing as a primary and secondary preventative approach. To get the hearing health message in mainstream media we partnered with the largest mobile operator in South Africa, Vodacom, as the main sponsor along with smaller sponsorships by the hearing industry (e.g. Sivantos, Oticon and the Ear Institute). Each sponsor purchased test credits that ensures a free hearing test can be offered to every South African on the hearZA app. In turn sponsors get an in-App advertisement right before the test commences indicating that their hearing test is sponsored. Every download offers three free hearing tests after which an inApp purchase option is available.

Along with the sponsors we invested in a strategic media campaign using the WHO's World Hearing Day as springboard from which to get the message out to the broadest possible audience. To support this effort we recorded four video's with local celebrities taking the hearZA test and who were willing to serve as hearing health ambassadors. These were disseminated through various social media platforms (Figure 1 example with >250 000 Facebook video views).

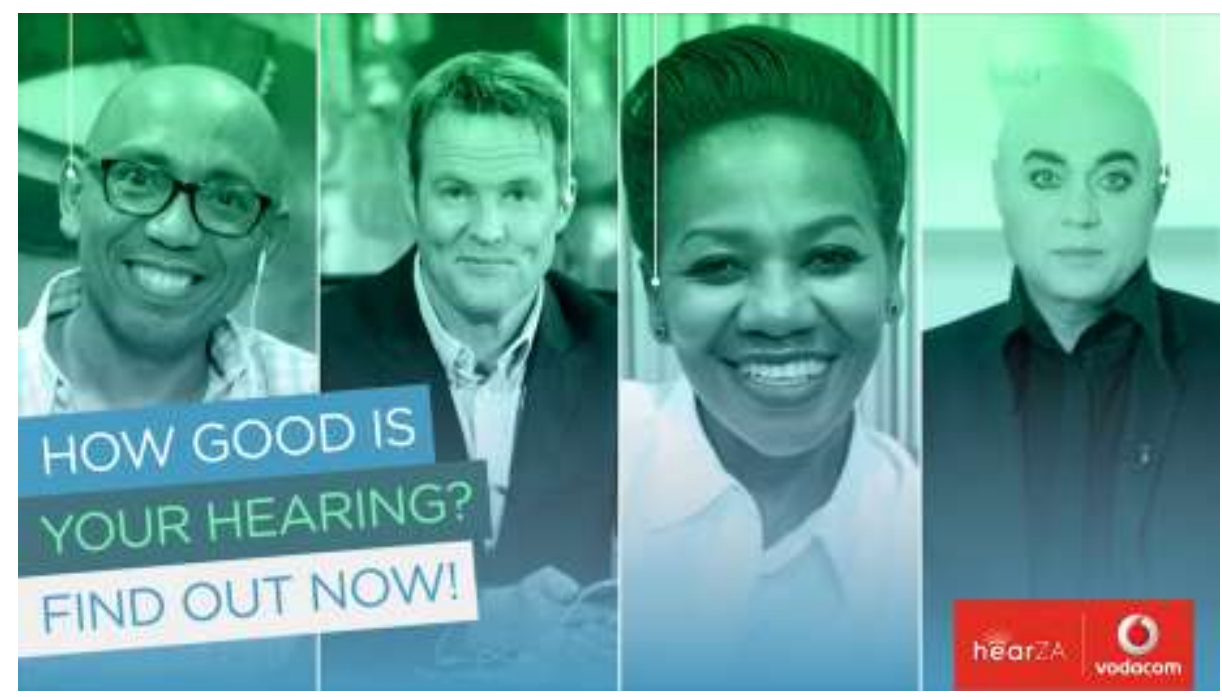

Figure 1. Video with local celebrity Nataniel taking the hearZA app test (www.youtube.com/watch?v=X274TjVyl8s)

The app also encourages sharing your hearing test through social media channels (with or without your score). Health messaging graphics for social media posts are provided as part of this sharing function (see figure 2) in which users can choose to 


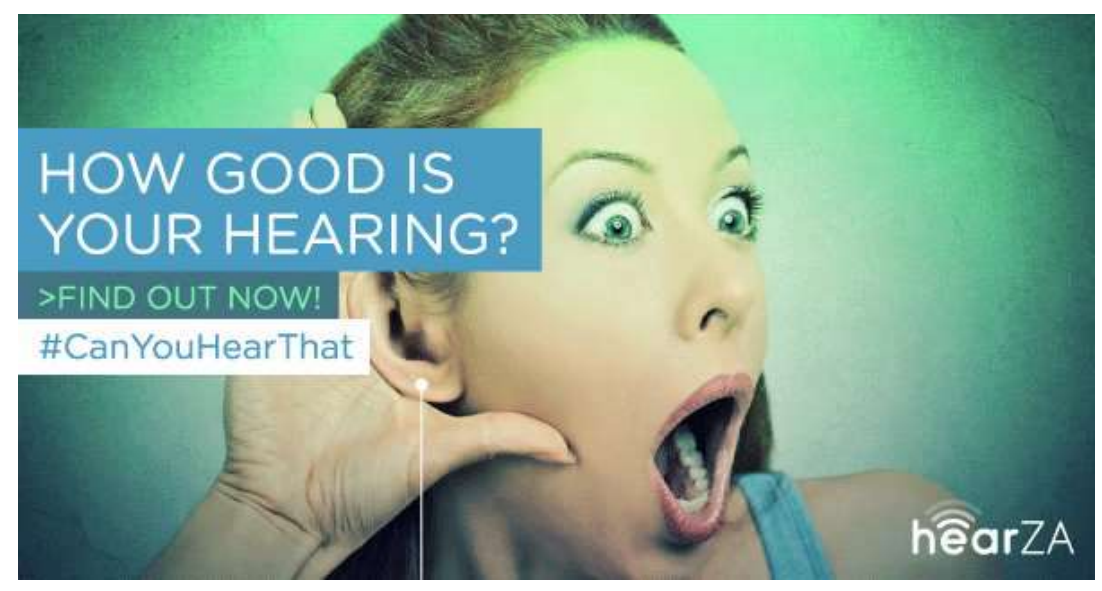

Figure 2. Example Twitter graphic vector for social media sharing.

share their personal hearing health score. The personalized score, deduced from the range of SNR's from our normative data set (Potgieter et al. 2016; Potgieter et al. Submitted), also allows a scoreboard feature encouraging users to track their score alongside facebook friends and VIP's who took the test. We also encourage participation through a \#CanYouHearThat campaign through radio and TV interviews and shows challenging persons to take and share the test through social media channels. These initiatives allowed us to reach a younger demographic with the peak number of persons tested between the age of 25 and 35 years.

\section{Accurate detection of hearing loss}

Test development was a partnership between audiology and computer engineering divisions at the University of Pretoria led by myself (De Wet Swanepoel) and Dr Herman Myburgh in collaboration with Dr Cas Smits from the VU Medical University Hospital in the Netherlands where digits-in-noise testing originated.

The binaural hearZA digits-in-noise test is validated for accurate testing across smartphones with no significant difference between headphone or earphone type (Potgieter et al. 2016). Test criteria are setup to identify best ear sensorineural hearing loss greater than $25 \mathrm{~dB} \mathrm{HL}$ (PTA 0.5, 1, 2 \& $4 \mathrm{kHz}$ ) with additional criteria for best ear sensorineural hearing loss greater than $40 \mathrm{~dB} \mathrm{HL}$ (PTA 0.5, 1, 2 \& $4 \mathrm{kHz}$ ). Test accuracy across native and non-native English speakers approximates $90 \%$ with sensitivity of $95 \%$ (Potgieter et al. Submitted). These values ensure a valid screening test that can be downloaded to end-user smartphones and conducted with any headphone or insert earbud. 


\section{Hearing health monitoring}

Smartphone health surveillance and monitoring has gained significant traction with an estimated 1.7 billion downloads expected by 2017 and global revenues reaching \$21.5 billion in 2018 (Economist, 2016). In line with the global trend of health monitoring on smartphones the hearZA app allows hearing health monitoring through a personal profile tracking the hearing health score over time. An in-app notification prompts users to retake the hearing test annually to ensure there has not been any significant changes in hearing health status. This hearing health monitoring feature can be personalized for different demographic groups with targeted preventative health messaging. For example, teenagers and young adolescents who are at a significantly increased risk of music-induced hearing loss may be targeted with messages around safe listening behaviors (WHO, 2015). This approach also ensures more general awareness around hearing health care, which has traditionally been a difficult and abstract health concept for the general public.

\section{Decision support tool for audiological intervention}

To support persons referred with the App we partnered with the Ida Institute to adapt a selection of their tools to provide in-app decision support in line with the principles of patient centered care. Help-seeking and hearing aid uptake can be seen as a behavioral change in adults with hearing loss. Integrating a brief decision-support tool, incorporating user-driven design, is offered as an optional 2-min guide to users who failed the hearZA test (Figure 3). The first tool to be incorporated is the "Why improve my hearing?" tool by the Ida Institute. The aim of the decision-support tool is to facilitate greater uptake of help-seeking by supporting the progression from more pre-contemplative and contemplative stages of change to action stages.

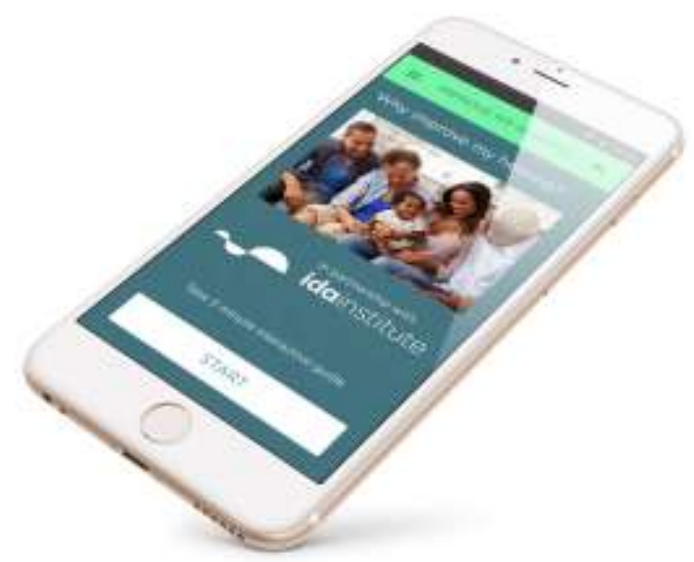

Figure 3. In-App decision-support tool developed with the Ida Institute 


\section{Referral linkage to audiologists}

The hearZA App was launched in partnership with the two professional organizations for audiologists in South Africa, the South African Association of Audiologists and South African Speech, Language and Hearing Association. Members of these societies were offered the opportunity to register their practices on the hearZA cloudbased management platform. Persons who fail a hearing test can look up their closest audiologist based on their geolocation within the App. A further feature allows persons who failed the test to request their closest audiologist to contact them directly. In this event the hearZA cloud-based management portal notifies the practice of the lead and requires audiologists to log in to the secure portal to view the contact details. This feature allows persons not only to be detected with a likely hearing problem but also links them directly to their closest providers.

\section{Conclusion}

A smartphone platform for a simple digits-in-noise based screening test lends itself to a whole range of far reaching and impactful opportunities for increased awareness, detection and uptake of hearing care. We found that the hearZA tool raised the public profile of hearing loss, the importance of healthy hearing and even of audiology in South Africa. We are partnering with associations to support similar initiatives in other countries. mHealth solutions like these provide new models of service-delivery and linkage to care that promise greater penetration, reach and even uptake of hearing health care.

\section{References}

Economist 2016, http://www.economist.com/news/business/21694523-mobilehealth-apps-are-becoming-more-capable-and-potentially-rather-useful-thingsare-looking).

Ericsson. Sub-Saharan Mobility Report. 2015 November, 2015.

Potgieter JM, Swanepoel D, Myburgh HC, Hopper TC, Smits C (2016). Development and validation of a smartphone-based digits-in-noise hearing test in South African English. International Journal of Audiology, 55(7):405-411

Potgieter JM, Swanepoel D, Myburgh HC, Smits C (2016). The South African English smartphone digits-in-noise hearing test: Effect of age, hearing loss and non-nativeness International Journal of Audiology, Submitted 
Statistics South Africa. 2013. General Houshold Survey. Available at: http://www.statssa.gov.za/publications/P0318/P03182013.pdf4 [last accessed 2015, May].

World Health Organization 2013. Global Estimates of Hearing Loss. Geneva: Switzerland. http://www.who.int/pbd/deafness/estimates/en/ World Health Organization 2015. Make Listening Safe. Geneva: Switzerland. http://www.who.int/pbd/deafness/news/safe_listening/en/ 